\title{
Méthode d'évaluation quantitative de l'hétérogénéité de la fracturation dans les carrières souterraines de calcaire à astéries
}

Université

1. allée Daguin

33607 Pessac Cedex Adrian.Cerepi@egid. u-bordeaux.fr
NDLE: Les discussions sur cet article sont acceptées jusqu'au 31 décembre 2002.
Durant ces dix dernières années, T'analyse quantitative de l'hétérogénéité de la fracturation par le Système d'information géographique (SIG) devient intéressante pour étudier la stabilité des carrières souterraines et des propriétés réservoirs induites par la fracturation et ses relations avec les écoulements souterrains. Cet article propose une méthode d'analyse quantitative de la fracturation du calcaire en carrières souterraines. Plus de 1000 fractures dans trois carrières souterraines carbonatées ont été relevées, mesurées, cartographiées et numérisées. L'analyse de l'hétérogénéité et de la connexité de la fracturation est fondée sur la répartition spatiale des paramètres géométriques de chaque fracture, tels que : ouverture, longueur, direction, orientation, écartement et distribution spatiale des familles de fractures.

Mots-clés : calcaire, réseau fissural, géométrie, carrières souterraines, réservoir.

\section{Quantitative evaluation method of fracturation heterogeneity in underground quarries of asteries limestone}

During the last decade the quantitative analysis of fracturation heterogeneity from Geographic Information System (GIS) has became very usefull for the modelling of underground quarries stability, the reservoir properties induced by fracturing and their consequence on ground water flow. This paper aims to give a method of quantitative analysis of fracture network in underground limestone quarries. More than 1,000 fractures taken from three limestone underground quarries were measured, mapped and numerized. The heterogeneity analysis and the fracturing connectivity are based on the spatial distribution of geometrical parameters of each fracture such as : openness, length, direction, orientation and on the spatial distribution of various fracture families.

Key words : limestone, fracture network, geometry, underground quarries, reservoir. 


\section{Introduction}

Cet article propose une méthode d'analyse quantitative de la fracturation du calcaire en carrières souterraines à partir de l'observation de trois carrières souterraines de Gironde (Saint-Germain-LaRivière, Thau et Maison-Franche). L'objectif est d'en montrer l'intérèt pour l'analyse de la stabilité des carrières souterraines et les possibilités qu'elle ouvre pour l'étude des propriétés réservoirs induites par la fracturation avec leurs conséquences sur la diagenèse (dissolution, sédimentation interne, cimentation), les propriétés pétrophysiques (porosité, perméabilité, transmissivité, capillarité) et les écoulements souterrains.

La méthode mise en œuvre, ici, pour l'analyse quantitative de l'hétérogénéité des caractères gêométriques du réseau de fractures comprend quatre étapes: la reconnaissance du contexte géologique, le relevé topographique des carrières souterraines, l'analyse géométrique in situ et le traitement numérique cartographique et statistique des données dans un SIG (Système d'information géographique) (Collet, 1992).

Ce travail est une contribution au problème de la modélisation des propriétés géotechniques et réservoirs des roches. Actuellement, la modélisation de l'état de fracturation d'un massif rocheux est fondée sur deux approches :

- la première utilise la représentation synthétique d'un réseau de fractures intégrant un ou plusieurs caractères géométriques qui, cependant, reste très éloignée de la réalité souvent plus complexe (Bourgine et al., 1998 ; Jourde et al., 1998). Toutefois, cette représentation synthétique est simplifiée ;

- la seconde concerne l'analyse quantitative, sur le terrain ou en forage, des caractères géométriques réels du réseau de fractures, tels que l'ouverture, l'orientation et l'espacement en utilisant des méthodes de traitement numérique d'image (Brière et Razack, 1982 : Delay et Bracq, 1993 ; Mania et al., 1998 ; Bodin et Razack, 1999).

Après un rappel succinct du contexte historique, géographique et géologique et des sites étudiés, nous présenterons successivement la morphologie des carrières souterraines, les caractères des fractures et les différentes étapes du traitement des données.

\section{2}

\section{Contexte historique, géographique et géologique}

\section{1}

\section{Contexte historique}

Entre le XVII" siècle et le début du XX $\mathrm{X}^{\mathrm{e}}$ siècle, le fort développement de l'urbanisation a entraîné une forte consommation de matériaux de construction. Il en est résulté l'ouverture de 2000 hectares de carrières souterraines réparties dans plus de 113 communes de la Gironde.

\section{Contexte géographique et géologique}

Les anciennes carrières souterraines d'exploitation de pierre de taille en Gironde sont localisées principalement dans l'Entre-Deux-Mers, sur la rive droite de la Dordogne et sur la rive droite de la Garonne. Elles ont été ouvertes dans le calcaire à astéries, formation de l'Oligocène supérieur (Stampien) du bassin d'Aquitaine, dénommée globalement "pierre de Bordeaux $n$.

Ce calcaire s'est formé durant la transgression de l'Oligocène supérieur $1 \mathrm{~b}$ au sein d'un système sédimentaire de plate-forme marginale constitué de quatre domaines de sédimentation : le domaine de sédimentation continentale à l'est (sables du Périgord et de l'Agenais), le domaine de sédimentation de transition (calcaire lacustre et marne sableuse), le domaine de sédimentation de plate-forme interne (calcaire à astéries) et le domaine de sédimentation marine ouverte sur le domaine océanique à l'ouest (marne) (Fig. 1).

Dans un environnement littoral soumis à l'action des vagues et des courants, le calcaire à astéries est constitué d'un sédiment bioclastique fin à grossier (algues, mollusques, coraux, échinodermes, foraminifères), grainstone à wackestone principalement, déposé sous forme de corps sédimentaires centimétriques à hectométriques emboités et/ou empilés les uns sur les autres. Cette accumulation hétérogène crée des structures sédimentaires du type lentilles, biseaux, chenaux, à stratification souvent oblique et entrecroisée, associées à des surfaces de discontinuités de changement de sédiment ou d'érosion, caractéristiques d'un environnement sédimentaire intertidal.

Depuis la fin de l'Oligocène jusqu'à aujourd'hui, dans les zones étudiées, le calcaire à astéries est resté émergé sous un très faible recouvrement alluvionnaire. La roche a été ainsi soumise à l'infiltration d'eaux météoriques depuis environ $32 \mathrm{Ma}$, créant ainsi en recouvrement des carrières souterraines une zone d'altération d'épaisseur métrique à décamétrique non exploitable. La forte hétérogénéité sédimentaire a été accentuée par la diagenèse créant des zones à forte dissolution des éléments de la roche et des zones plus ou moins bien cimentées par de la calcite (Cerepi, 1997). La dissolution associée aux zones à forte perméabilité, sédimentaire ou acquise par la fracturation, a permis le développement d'un réseau karstique.

La fracturation est liée aux principales structures de déformation souple et cassante des terrains tertiaires créés par l'orogenèse pyrénéenne (Pratviel, 1972; Gayet, 1980; Beaufrère, 1986) (Fig. 2). L'analyse du réseau de fractures et des réseaux karstiques effectuée dans différentes carrières de la Gironde met en évidence une néotectonique post-Oligocène appartenant aux deux directions principales armoricaine et varisque, associées à l'orogenèse hercynienne (Beaufrère, 1986; Cerepi, 1997):

- les accidents de direction armoricaine NW-SE : faille de la Garonne qui dessine le cours de la Garonne, le synclinal de Bourg-sur-Gironde et le synclinal de Cavignac-Villegouge ;

- les accidents de direction varisque NW-SE sont moins développés. 


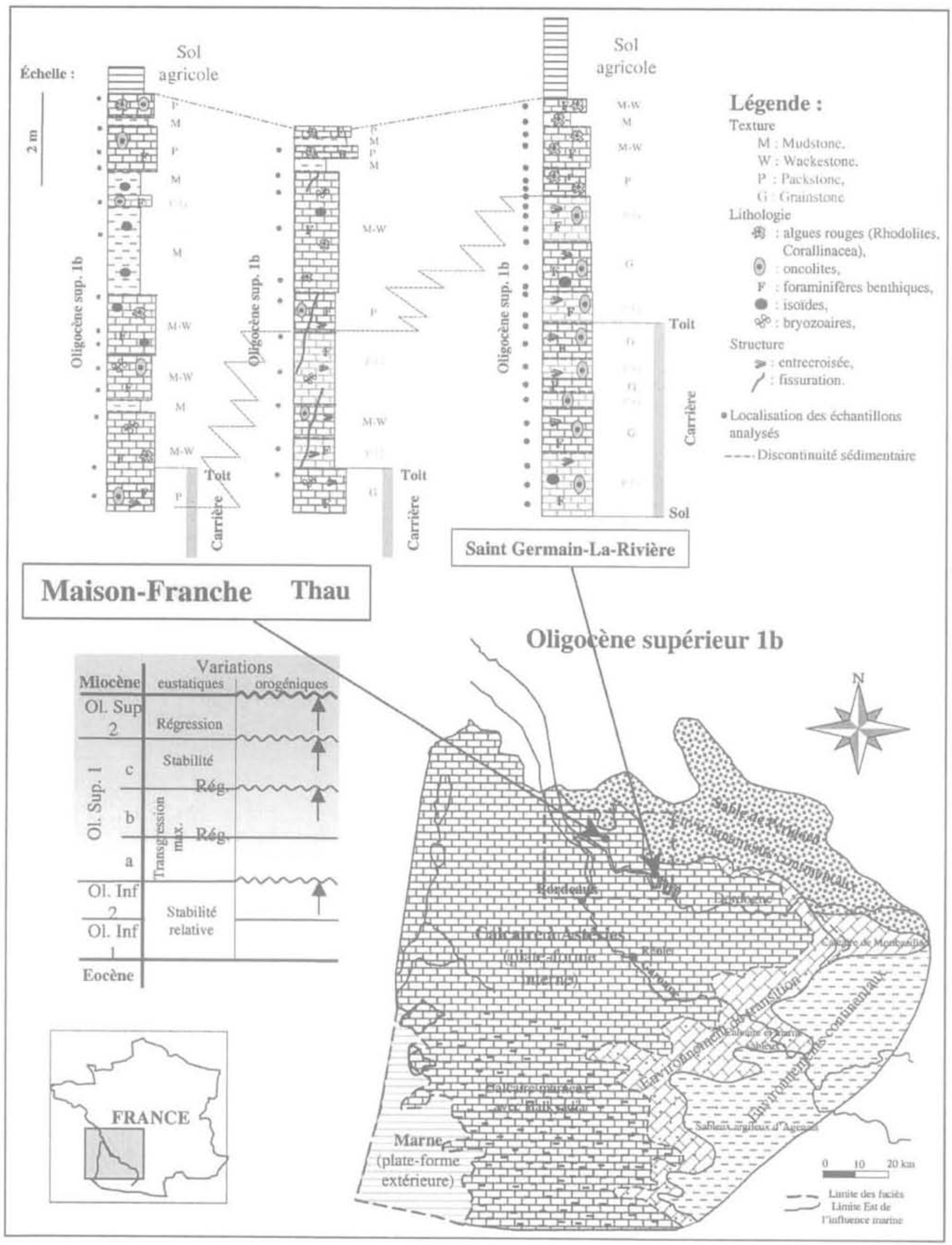

FlG.1 Localisation des carrières de Gauriac $(x=366,4 ; y=312,3)$ et de Saint-Germain-La-Rivière $(x=390,3$; $y=298,3$ ), leur log lithostratigraphique et le contexte sédimentaire au nord du bassin d'Aquitaine durant I'Oligocène supérieur 1b (Pratviel, 1972; Gayet, 1980 ; Cerepi, 1997).

Localization of Gauriac quarrie $(x=366.4 ; y=312.3)$ and Saint-Germain-La-Rivière quarrie $(x=390.3 ; y=298.3)$, their lithostratigraphic log and the sedimentary context in the North of Aquitaine during the Upper Oligocene $1 \mathrm{~b}$ (Pratviel, 1972; Gayet, 1980; Cerepi, 1997). 


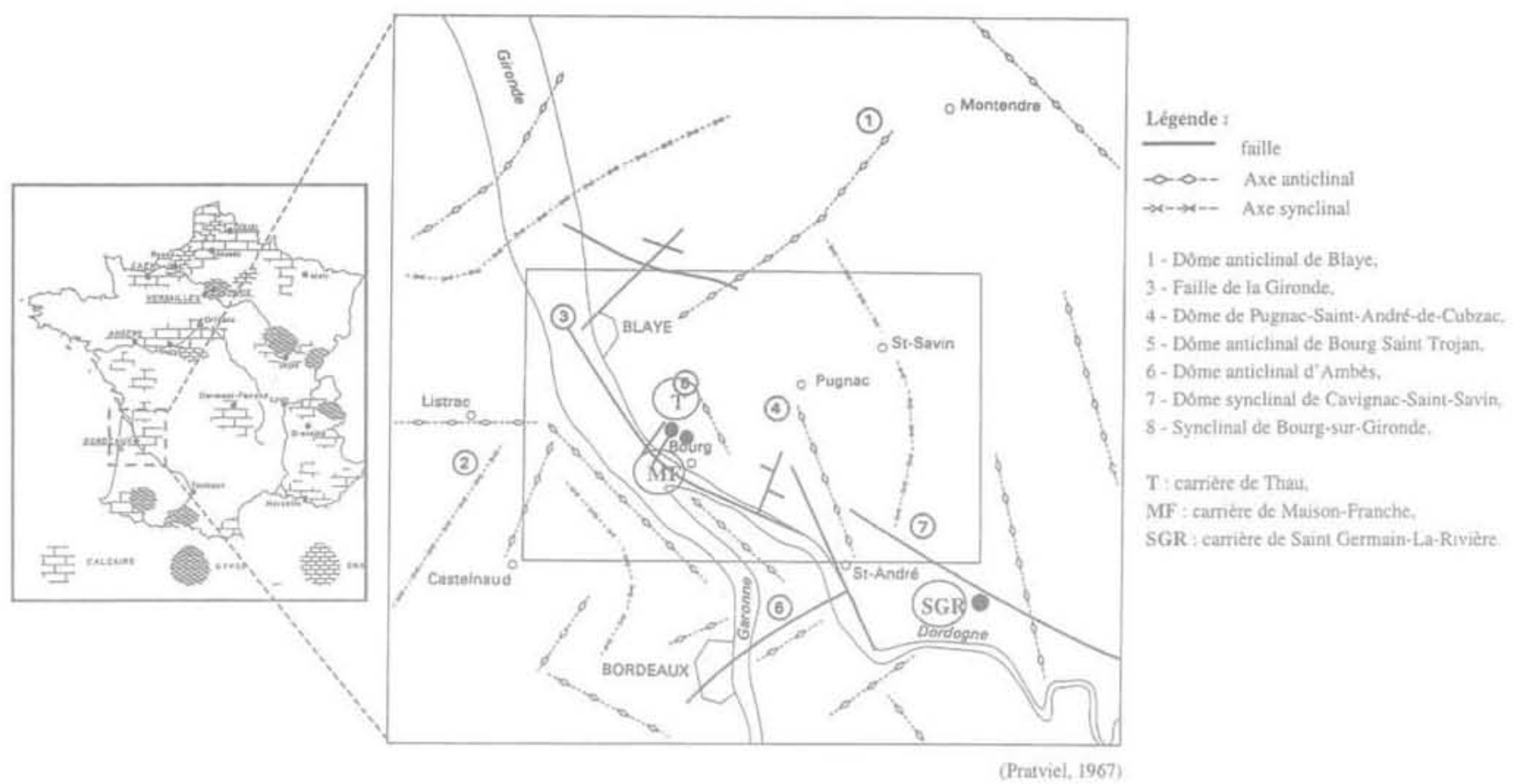

F0.2 Contexte structural du Nord du bassin d'Aquitaine.

Structural setting of North Aquitaine Basin.

\section{3}

\section{Morphologie des carrières étudiées}

La morphologie des trois carrières souterraines étudiées est marquée par la méthode d'extraction par "chambres et piliers abandonnés ». La dimension et la répartition des galeries et des piliers permet de calculer le taux de défruitement. L'épaisseur du recouvrement varie entre $3 \mathrm{~m}$ et $10,9 \mathrm{~m}$. La contrainte verticale régnant dans les piliers est calculée par la méthode de l'aire tributaire (Allard, 1975) (tableau 1).

Dans la carrière de Saint-Germain-La-Rivière, les galeries principales, les plus longues, sont orientées est-ouest ; les galeries secondaires, plus courtes, nordsud. Le taux de défruitement moyen est de $63 \%$ et l'épaisseur du recouvrement varie entre $3,4 \mathrm{~m}$ et $4,2 \mathrm{~m}$.

Dans la carrière de Maison-Franche, les galeries principales sont orientées $\mathrm{N} 130^{\circ}$; les galeries secondaires $\mathrm{N} 30^{\circ}-\mathrm{N} 40^{\circ}$. Le taux de défruitement, de $70,6 \%$ est très élevé, et l'épaisseur du recouvrement varie de $4,62 \mathrm{~m}$ à l'est à $10,86 \mathrm{~m}$ à l'ouest.

Enfin, la carrière de Thau présente cinq niveaux d'exploitation dont seul le niveau II, à partir de la surface, a été étudié. Les galeries principales sont orien- tées $\mathrm{N} 130^{\circ}$, les galeries secondaires $\mathrm{N} 30^{\circ}-40^{\circ}$. Le taux de défruitement est de $64,4 \%$. L'épaisseur du recouvrement augmente progressivement d'ouest $(8,7 \mathrm{~m}) \mathrm{en}$ est $(14,45 \mathrm{~m})$.

\section{4}

\section{Caractérisation des discontinuités}

\section{1}

\section{Fractures}

Dans les trois sites étudiès, les fractures sont soit d'origine tectonique (fractures anciennes dites " géologiques $n)$ soit d'origine anthropique, liées à l'exploitation des carrières (fractures récentes dites «mécaniques $n$ ). Les fractures géologiques ont une ouverture de 1 à $200 \mathrm{~mm}$ et sont colmatées par des sédiments internes argileux ou argilo-sableux rouges ou verdảtres remaniés depuis la surface. Souvent, leurs épontes sont colorées et présentent des traces de dissolution (karstification). Les fractures mécaniques ont une ouverture inférieure à $1 \mathrm{~mm}$, avec un rapport ouverture/longueur

TABLEAUI Caractéristiques géométriques des trois carrières souterraines étudiées. Geometrical parameters of three studied underground quarries.

\begin{tabular}{|c|c|c|c|c|c|c|c|c|c|c|c|c|c|}
\hline \multirow[t]{2}{*}{ Sites étudiès } & \multicolumn{3}{|c|}{$\begin{array}{c}\text { Epaisseur } \\
\text { du recouvrement }(\mathrm{m}) \\
\end{array}$} & \multicolumn{3}{|c|}{$\begin{array}{c}\text { Hauteur } \\
\text { des piliers (m) }\end{array}$} & \multicolumn{3}{|c|}{$\begin{array}{c}\text { Section des piliers } \\
\left(\mathrm{m}^{2}\right)\end{array}$} & \multirow{2}{*}{$\begin{array}{l}\text { Taux de } \\
\text { défruitement } \\
(\%)\end{array}$} & \multicolumn{3}{|c|}{$\begin{array}{c}\text { Contrainte verticale } \\
\text { statique (Mpa) }\end{array}$} \\
\hline & min & moy & $\max$ & $\min$ & moy & $\max$ & min & moy & $\max$ & & min & moy & $\max$ \\
\hline $\begin{array}{l}\text { Saint-Germain- } \\
\text { La Rivière }\end{array}$ & 3.0 & 4.4 & 7,2 & 2,1 & 3.2 & 4,4 & 6.6 & 19,1 & 69,9 & 63.0 & 0,2 & 0,3 & 0,5 \\
\hline Thau & 8.7 & 11,5 & 14,5 & 1,0 & 1,6 & 2,4 & 4,2 & 31,0 & 116,5 & 64,3 & 0,3 & 0,6 & 1,0 \\
\hline Maison-Franche & 4,6 & 8,6 & 10,9 & 0,6 & 1,4 & 2,6 & 4,5 & 18,9 & 127.8 & 70,6 & 0,2 & 0,5 & 1,2 \\
\hline
\end{tabular}


très élevé $(>0,005)$. Leurs épontes, bien tranchées et parallèles entre elles, ne présentent pas ou peu de traces de dissolution.

Assez fréquemment, des fractures géologiques colmatées sont réouvertes avec apparition de fractures dans le colmatage ou avec décollement de celui-ci des épontes. Les observations mettent en évidence deux cas de figures (Fig. 3):

- soit la fracture mécanique évolue parallèlement à la direction de la fracture géologique:

- soit la fracture mécanique est transverse à la fracture géologique.

\section{0}

\section{Discontinuités}

Des karsts transverses et stratiformes apparaissent au toit des galeries et à travers les piliers. Les cavités et chenaux de dissolution karstique sont de dimension centimétrique à métrique (Fig. 3). Les karsts sont la plupart du temps colmatés par des produits argileux rouges ou verdảtres et/ou un sédiment argilo-sableux ou sablo-argileux à stratification oblique lenticulaire ou chenaux. Les karsts stratiformes sont des cavités issues de la dissolution et disposées subparallèlement à la stratification du calcaire. Ils se développent, préférentiellement, dans les surfaces de discontinuités sédimentaires lesquelles délimitent des

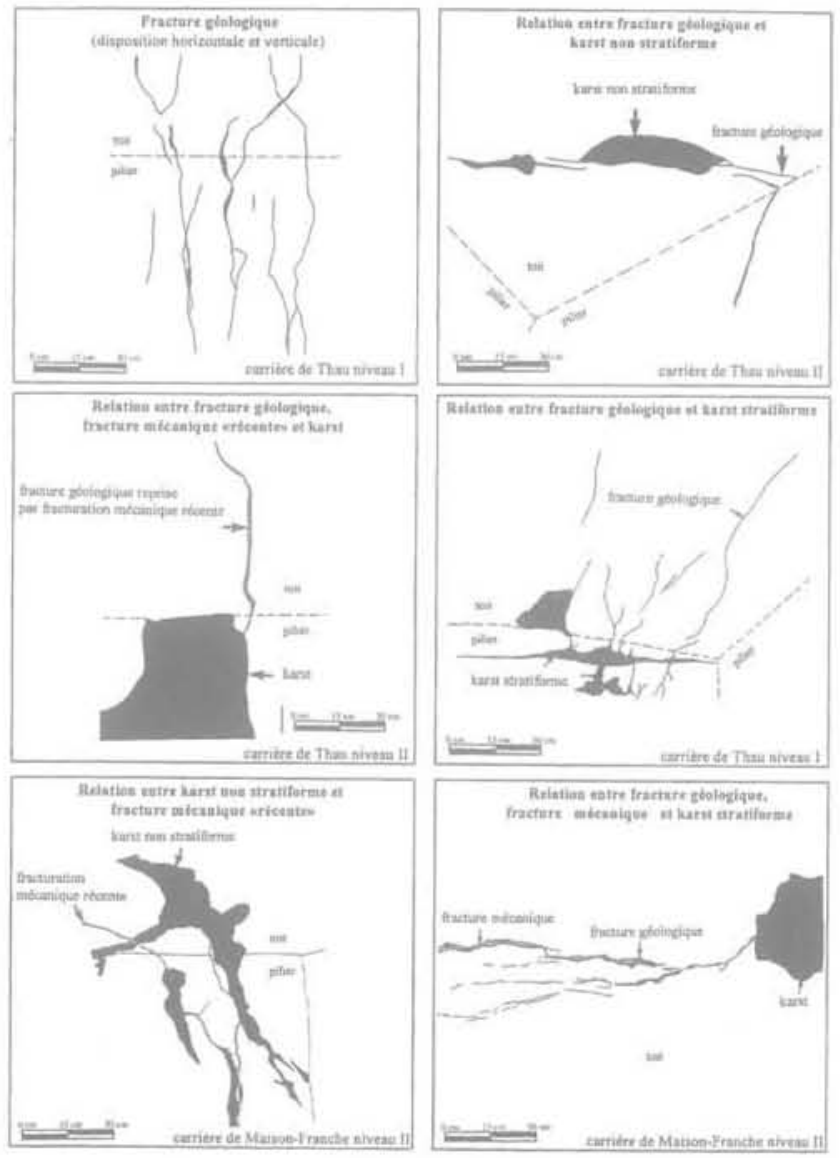

FIG,3 Typologie et relations observées entre les fractures géologiques, les fractures mécaniques et les karstifications dans les sites étudiés.

Types and relationships observed between geological fracture, mechanical fracture and karstification in different studied sites. corps sédimentaires d'extension horizontale métrique à décamétrique et de granulométrie grossière (calcirudite). Les karsts transverses se développent transversalement par rapport à la stratification. Ils sont aussi, généralement, colmatés par les mèmes types de sédiments.

\section{5}

\section{Données et traitement des données}

- Acquisition et pré-traitement. Sur le terrain, la position, la longueur apparente et la direction sont relevées sur un plan topographique au 1/200. Représentée par un segment de droite $S\left(x_{1}, y_{1}\right.$ et $\left.x_{2}, y_{2}\right)$, chaque fracture est définie dans le plan d'observation (toit de la carrière) par les coordonnées de son barycentre $\mathrm{g}\left(\mathrm{x}_{\mathrm{b}}\right.$, $y_{0}$ ) (Fig. 4). Pour utiliser les données sur carte topographique, il est nécessaire de géoréférencer le plan
(A)

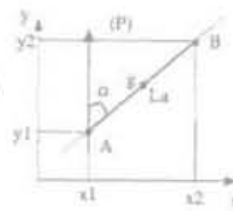

(B)

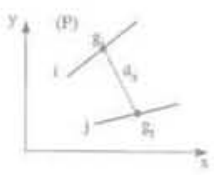

(D)

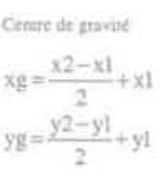

$$
\begin{aligned}
& x g=\frac{x 2-x 1}{2}+x 1 \\
& y g=\frac{y 2-y !}{2}+y 1
\end{aligned}
$$

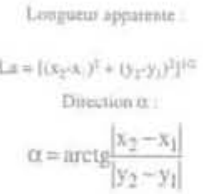

(C)

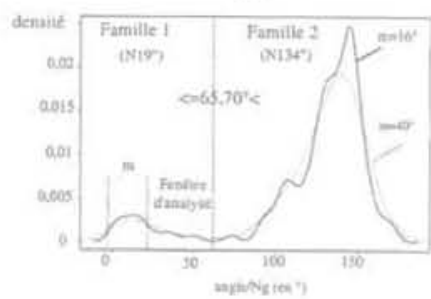

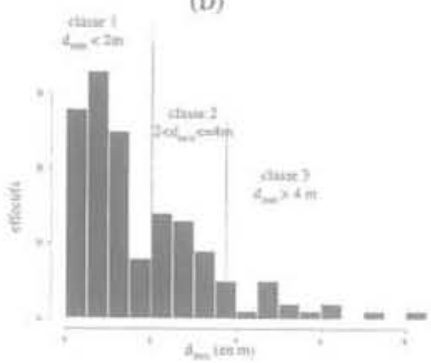

(E)

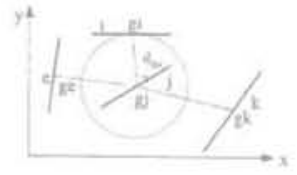

FiG.4 Différents paramètres géométriques utilisés dans l'analyse du système de fractures :

A) Définition de la position des fractures dans le plan de la carrière.

B) Distance entre deux fractures d dans les carrières.

C) Définition des familles de fractures par la méthode de $\alpha$ fenètre mobile $x$.

D) Exemple d'histogramme de distance $\mathrm{d}_{\min }$ et définition des trois classes de compacité.

E) Calcul des plus proches fractures voisines. Different geometrical parameters used in the analysis of fracturing network :

A) Definition of fractures localization in quarry map.

B) Distance between two fractures $d_{j}$ in quarries.

C) Definition of fractures classes by the "moving window method.

D) Example of distance histogram $d$ and the definition of three density classes.

E) Calculation of the most fractures neighbouring. 
d'observation par rapport au système de coordonnées (Lambert III pour les cartes IGN utilisées). Pour passer du système de coordonnées de la table à digitaliser à un système de coordonnées géoréférencées, les données subissent des transformations géométriques SIG (Collet, 1992). L'estimation des fonctions de transformation est réalisée par la technique des moindres carrés appliquée sur au moins trois points de contrôle ou " amers ») localisés dans les deux systèmes. La transformation utilisée dans le cas présent est du type affine et comprend trois coefficients: transformation simple de translation, transformation par changement d'échelle et transformation par rotation. La résolution des transformations obtenues lors du traitement est de $0,1 \mathrm{~m}$ : les calculs effectués sont à $\pm 0,1 \mathrm{~m}$ près.

- Représentativité des fractures analysées, Le nombre de fractures analysé doit être représentatif pour la surface considérée. Pour ce faire, nous avons comparé une distribution expérimentale de points voisins (centre de gravité des fractures) à une distribution poissonnienne (Collet, 1992). Le coefficient de représentativité Rs est donné alors par la relation (1) :

$$
\mathrm{Rs}=\frac{\sqrt{\mathrm{n}} \cdot \sum_{\mathrm{i}=1}^{\mathrm{n}} \text { Dobs }_{\mathrm{i}}}{0,5 \cdot \mathrm{n} \cdot \sqrt{\mathrm{S}}}
$$

où $n$ est le nombre de fractures ; $S\left(\mathrm{~m}^{2}\right)$ est la surface des carrières étudiées; Dobs ( $m$ ) est la distance entre le point $i$ et son plus proche voisin. Les valeurs calculées de Rs pour les carrières étudiées sont supérieures à l'unité, ce qui tendrait à montrer que le nombre de fractures étudiées (plus de 1000 ) est suffisant par rapport aux surfaces des carrières (environ $10000 \mathrm{~m}^{2}$ ).

- Paramètres géométriques. Plusieurs paramètres géométriques ont été calculés (Fig. 4). La longueur apparente d'une fracture ( $\mathrm{La}$ ) est la lonqueur du segment de droite correspondant à l'intersection du plan de la fracture et du plan d'observation (toit de la carrière). La direction $\alpha$ d'une fracture représentée par un segment de droite $S(x, y$, et $x, y)$, est donnée par rapport au nord géographique. Les familles de fractures ont été définies, par rapport à leur direction, par la méthode de l'estimation de la densité de "fenêtre mobile» (Saporta, 1991). Elle consiste à construire une classe de longueur $m$ autour de $x$ ( $1 x=[x-m / 2$; $x+m / 21)$. On compte alors le nombre d'observations $p$ appartenant à cette classe. On estime la distribution $f(x)$ par le rapport $p / p$. Elle permet de construire point par point la fonction de densité $f(x)$. Afin d'avoir une densité symétrique, nous avons utilisé la distribution gaussienne comme fonction d'estimation de densité symétrique, La taille de la fenêtre $m$ est choisie pour s'assurer de la stabilité des familles de fractures observées et pour détecter les familles secondaires incluses dans les familles principales (Fig. 4).

La densité des fractures peut être appliquée soit à des fractures quelconques, soit à des fractures de même famille directionnelle. Deux définitions de densité de fractures sont utilisées dans cette étude. On définit la densité surfacique et la densité linéaire. La densité surfacique $D$. est exprimée en nombre $N$ de fractures i observées dans le plan d'observation par rapport à l'unité de surface :

$$
\mathrm{D}_{\mathrm{si}}=\frac{\mathrm{N}_{1}}{\mathrm{~S}_{1}}
$$

où $S\left(\mathrm{~m}^{2}\right)$ est la surface totale de la zone étudiée dans le plan d'observation. La densité linéaire $D_{4}$ par unité de surface $\left(m^{2}\right)$ est définie par:

$$
\mathrm{D}_{\sharp}=\frac{\mathrm{La}_{i}}{\mathrm{~S}_{i}}
$$

où $\mathrm{La}(\mathrm{m})$ est la longueur apparente cumulée des fractures observées dans le plan d'observation. La fréquence des fractures $\mathrm{F}$ est définie comme :

$$
F_{i}=\frac{N}{N}, 100
$$

où $N$ est le nombre de fractures de la famille $i ; N$ est le nombre total des fractures.

- Compacité des fractures. Dans le plan d'observation, les fractures sont plus ou moins serrées les unes par rapport aux autres. Dans le cas des fractures parallèles, l'écartement (e) définit la distance qui sépare deux fractures voísines parallèles (Ruhland, 1973 ; Dutartre, 1982; Blès et Feuga, 1982). Étant donné $n$ fractures $i$ (de mème famille directionnelle) et leur écartement moyen e, la compacité C. $\left(\mathrm{m}^{-1}\right)$ est définie par la relation :

$$
C_{i}=\frac{1}{\bar{e}_{i}}
$$

où e : écartement moyen (m).

Dans le cas des fractures non parallèles, étant donné deux fractures i et j non parallèles, leur écartement est la distance $d_{i}$ entre leurs barycentres $g_{\text {i }}$ et $g_{j}$ : pour $n$ fractures $i$ et $j$, il est nécessaire de calculer la

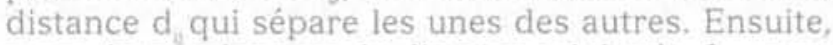
pour chaque fracture, la distance minimale $\mathbf{d}_{\min }$ avec la fracture la plus proche est calculée (Figs. 4D, E). Trois classes de compacité sont définies à l'aide de I'histogramme de distribution des classes de $\mathrm{d}$ d'égale distance : la classe 1 de compacité forte $\left(0<\mathrm{d}_{1}\right.$ $<=2 \mathrm{~m})$; la classe 2 de compacité moyenne $\left(2 \mathrm{~m}<\mathrm{d}_{2}<\right.$ $=4 \mathrm{~m}$ ) et la classe 3 de compacité faible $(4 \mathrm{~m}<\mathrm{d}$ ). A partir de ces classes, on trace les cartes d'isocompacité des fractures (cartes d'isodistance minimale d des fractures). Ensuite, les classes de compacité permettent de localiser les zones des carrières oủ les fractures ont une compacité située dans ces classes. Les résultats sont présentés sous la forme : de cartes de compacité de fractures et de tableaux analytiques des valeurs des paramètres extraites des cartes de compacité (Fig. 9)

- Connexité d'une fracture. Étant donné une fracture i, sa connexité est égale aux nombres de fractures i, j, k, l, n qu'elle recoupe (Fig. 5) :

- la connexité est égale à 0 si la fracture i est isolée ; - la connexité est égale à 1 si la fracture i est sécante à une seule fracture $j$;

- la connexité est égale à 2 si la fracture i est sécante à deux fractures $\mathrm{j}$ et $\mathrm{k}$;

- la connexité est égale à 3 si la fracture i est sécante à trois fractures j, $k$ et l ;

- la connexité est égale à n si la fracture i est sécante à n fractures j, k, 1, ..., n.

- Algorithme de calcul de la connexité. Si l'on considère deux fractures i et j représentées par deux segments Si $\left(x_{1}^{j}, y_{1}^{j}, x_{2}^{j}, y_{2}^{j}\right)$ et $S_{j}\left(x_{1}^{j}, y_{1}^{j}, x_{2}^{j}, y_{2}^{j}\right)$ et à partir de leurs équations il est possible de calculer les coordonnées xs et ys de leur intersection. Les segments de 


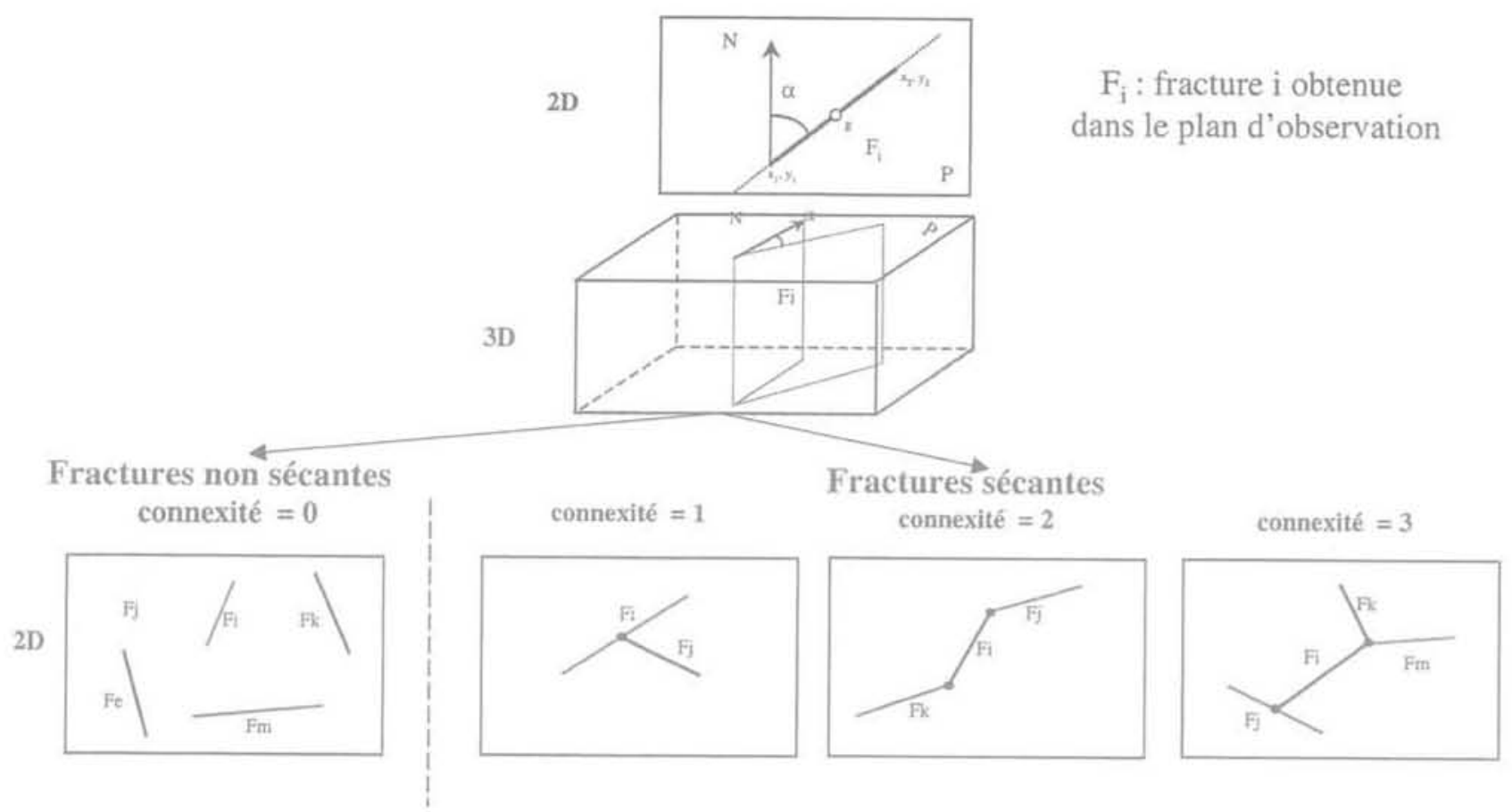

FiG.5 Définition de la connexité des fractures, fractures sécantes et non sécantes.

Definition of the fracture connectivity, connected fractures and non connected fractures.

droite représentant les fractures $i$ et $j$ sont définis par (6) et (7):

$$
\text { (6) } \frac{y-y_{1}^{1}}{x-x_{1}^{1}}=\frac{y_{1}^{1}-y_{2}^{1}}{x_{1}^{1}-y_{2}^{1}} \quad \text { et } \quad \frac{y-y_{1}^{1}}{x-x_{1}^{j}}=\frac{y_{1}^{j}-y_{2}^{j}}{x_{1}^{j}-y_{2}^{j}}
$$

Les coordonnées du point d'intersection $\left(\mathrm{x}_{\mathrm{s}}, \mathrm{y}_{\mathrm{s}}\right)$ entre les segments de droite représentant les fractures i et $\mathrm{j}$ sont définies par la relation suivante :

$$
\begin{aligned}
& x_{s}=\frac{1}{\Delta}\left[\left(x_{1}^{j} y_{2}^{j}-x_{2}^{j} y_{1}^{j}\right)\left(x_{1}^{j}-x_{2}^{j}\right)-\left(x_{1}^{j} y_{2}^{j}-x_{2}^{j} y_{1}^{j}\right)\left(x_{1}^{j}-x_{2}^{j}\right)\right] \\
& y_{s}=\frac{1}{\Delta}\left[\left(x_{1}^{j} y_{2}^{j}-x_{2}^{j} y_{1}^{j}\right)\left(y_{1}^{j}-y_{2}^{j}\right)-\left(x_{1}^{j} y_{2}^{j}-x_{2}^{j} y_{1}^{j}\right)\left(y_{1}^{\dagger}-y_{2}^{j}\right)\right]
\end{aligned}
$$

avec $\Delta=\left|\begin{array}{l}y_{1}^{\prime}-y_{2}^{\prime} x_{1}^{\prime}-x_{2}^{\prime} \\ y_{1}^{\prime}-y_{2}^{\prime} x_{1}^{\prime}-x_{2}^{\prime}\end{array}\right|$

Le point d'intersection $\left(x_{0}, y_{s}\right)$ se situe à la fois sur les deux fractures (Fig. 5). Ensuite, les fractures sont classées en fractures non sécantes (connexité nulle) et en fractures sécantes (connexité supérieure ou égale à 1). Ainsi, il est possible de calculer pour chaque carrière, le nombre et la connexité des fractures non sécantes et sécantes, leur fréquence respective, la longueur apparente moyenne, leur densité par unité de surface et d'établir une carte de compacité.

\section{6}

\section{Résultats}

Les réseaux de fractures à Thau niveau II et MaisonFranche niveau II présentent les mêmes caractéristiques géométriques et appartiennent au même système de fractures. En effet, les fractures géologiques et mécaniques présentent deux familles directionnelles : famille $1\left(\mathrm{~N} 19^{\circ}\right)$ et famille $2\left(\mathrm{~N} 131^{\circ}-134^{\circ}\right)$ (tableau II, Figs. 6, 7). La famille 2 est prédominante et fortement karstifiée, elle souligne que la direction SE-NW (accidents de direction armoricaine) est également une direction de drainage karstique lors de l'émersion post-oligocène du calcaire. Dans les zones où la compacité des fractures est faible, la longueur apparente moyenne des fractures est plus grande (elle passe de 2,26 m à 2,45 m) (Fig. 7). La longueur apparente moyenne des fractures de cette famille 2 est plus élevée que celle des fractures de la famille 1.

Le réseau de fractures à Saint-Germain-La-Rivière présente des caractéristiques complètement différentes avec 4 familles directionnelles dont deux prédominantes : famille $2\left(\mathrm{~N}^{\circ} 2^{\circ}\right)$ et famille $3\left(\mathrm{~N} 103^{\circ}\right)$ (tableau II, Figs, 6, 7).

\section{Discussions}

\section{1}

\section{Relation entre les familles de fractures et le contexte tectonique régional}

A partir des données d'observations en carrières souterraines il n'a pas été possible de construire un tectonogramme permettant d'analyser la cinématique des familles de fractures associées aux failles et aux plis lors des mouvements tectoniques post oligocène. Seules les cartes de fractures, les cartes de compacité des fractures des différentes familles et des cartes de compacité totale des fractures sont utilisables pour rechercher des relations entre la direction des fractures locales et les directions des plis régionaux. 
Maison Franche niveau II

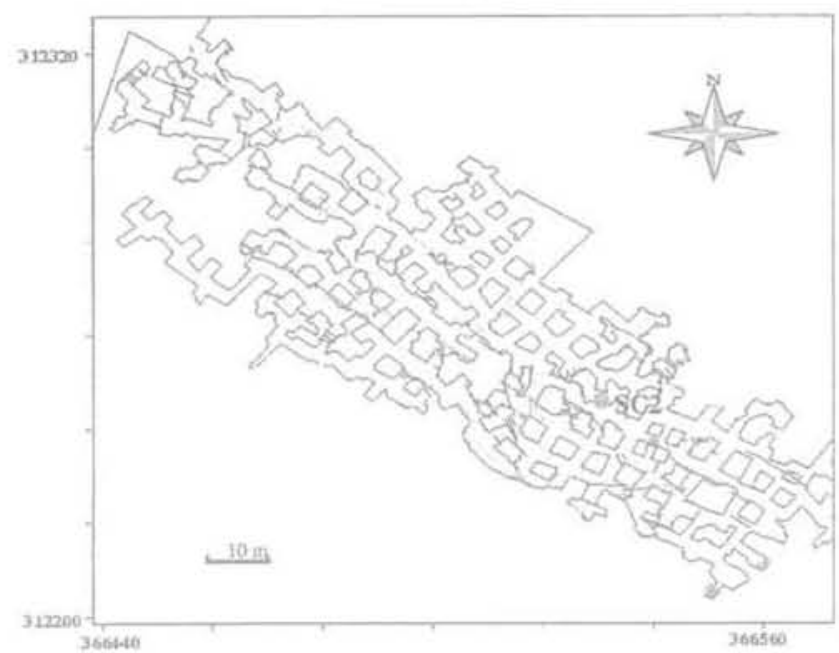

Fréquence (en \%)

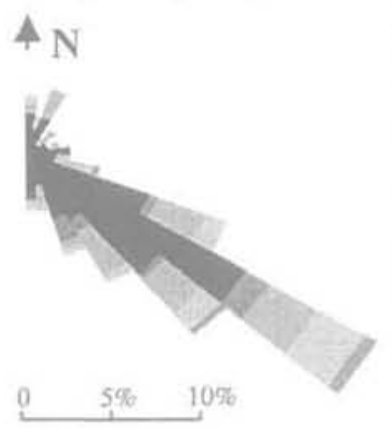

Longueur moyenne des segments (m)

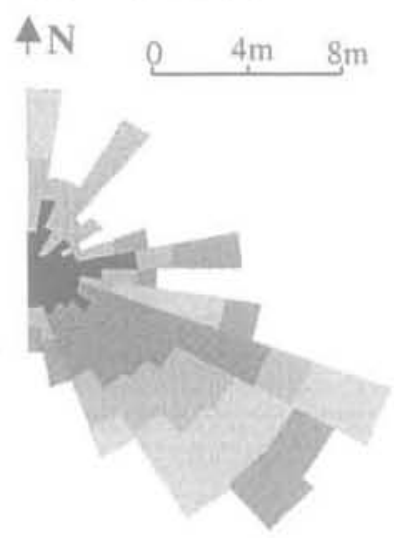

Fréquence (en \%)

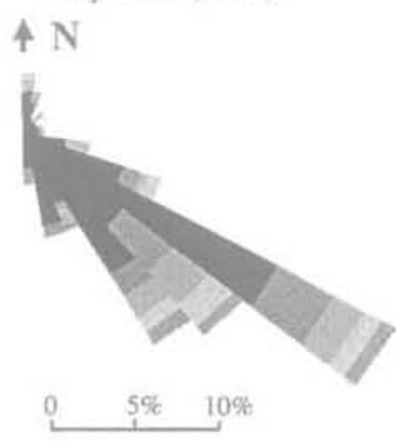

Frequence (en $\%)$

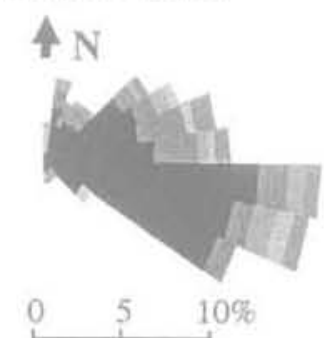

Longueur moyenne des fractures (en m)

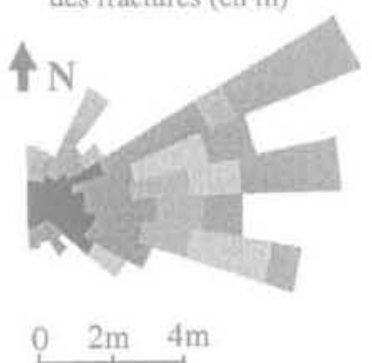

Légende :

\begin{tabular}{|ll}
\hline sondage caroté : & fracture mécanique superposée \\
fracture géologique: & a fracture géologique: \\
fracture géologique \& & karsi non strutiforme : \\
karst non stratiforme: & karst stratiforme avec ou \\
fracture mécanique : & $\begin{array}{l}\text { sans argiles: } \\
\text { pilier }\end{array}$
\end{tabular}

FlG. 6 Répartition et caractères des fractures dans les trois sites étudiés. Distribution and characters of fractures in three studied sites. 
TABLEAUIII Les caractéristiques géométriques des fractures dans les trois sites étudiés. Geometrical parameters of fractures in three studied sites.

\begin{tabular}{|c|c|c|c|c|c|c|c|}
\hline Sites & Familles & Paramètres & Minimum & Moyenne & Maximum & Écart-type & $\mathrm{Nb}$, de fract. \\
\hline \multirow{2}{*}{$\stackrel{巳}{\underline{E}}$} & 嵌市 & $\begin{array}{l}\text { écartement efm) } \\
\text { compacité } 1 / \mathrm{e}\left(\mathrm{m}^{-1}\right) \\
\text { longueur }(\mathrm{m}) \\
\text { orientation }(\%)\end{array}$ & $\begin{array}{l}0,00 \\
0,40 \\
0,00 \\
\mathrm{~N} 1\end{array}$ & $\begin{array}{l}0,50 \\
8,50 \\
2,30 \\
\text { N18 }\end{array}$ & $\begin{array}{r}2,80 \\
51,70 \\
9,60 \\
\mathrm{~N} 52\end{array}$ & $\begin{array}{r}0,60 \\
13,80 \\
1,30 \\
14,43\end{array}$ & 24 \\
\hline & 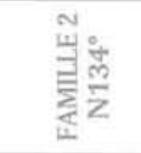 & $\begin{array}{c}\text { écartement } \mathrm{e}(\mathrm{m}) \\
\text { compacité } 1 / \mathrm{e}\left(\mathrm{m}^{-1}\right) \\
\text { longueur }(\mathrm{m}) \\
\text { orientation }\left({ }^{\circ}\right)\end{array}$ & $\begin{array}{l}0,10 \\
0,30 \\
0,50 \\
N 72\end{array}$ & $\begin{array}{r}0,40 \\
31,20 \\
1,50 \\
\mathrm{~N} 134\end{array}$ & $\begin{array}{r}3,10 \\
548,50 \\
5,20 \\
N 174\end{array}$ & $\begin{array}{r}0,60 \\
108,20 \\
1,10 \\
18,06\end{array}$ & 230 \\
\hline \multirow{2}{*}{ 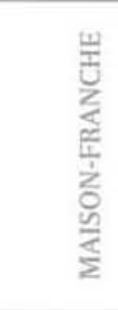 } & 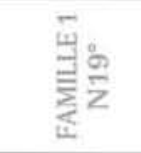 & $\begin{array}{l}\text { écartement e (m) } \\
\text { compacité } 1 / \mathrm{e}\left(\mathrm{m}^{-1}\right) \\
\text { longueur }(\mathrm{m}) \\
\text { orientation }\left({ }^{\circ}\right)\end{array}$ & $\begin{array}{l}0,32 \\
0,12 \\
0,29 \\
\text { No,7 }\end{array}$ & $\begin{array}{c}1,91 \\
1,28 \\
1,74 \\
N 19,07\end{array}$ & $\begin{array}{c}8,45 \\
3,16 \\
5,25 \\
N 42,59\end{array}$ & $\begin{array}{r}2,12 \\
1,00 \\
1,12 \\
14,00\end{array}$ & 28 \\
\hline & 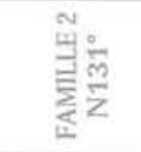 & $\begin{array}{l}\text { écartement e }(\mathrm{m}) \\
\text { compacité } 1 / \mathrm{e}\left(\mathrm{m}^{-1}\right) \\
\text { longueur }(\mathrm{m}) \\
\text { orientation }\left({ }^{\circ}\right)\end{array}$ & $\begin{array}{l}0,10 \\
1,56 \\
0,57 \\
N 59\end{array}$ & $\begin{array}{r}0,10 \\
56,24 \\
2,35 \\
\text { N130 }\end{array}$ & $\begin{array}{r}0,64 \\
1383,00 \\
16.43 \\
N 178\end{array}$ & $\begin{array}{r}0,10 \\
150,36 \\
1,74 \\
18,00\end{array}$ & 225 \\
\hline \multirow{4}{*}{ 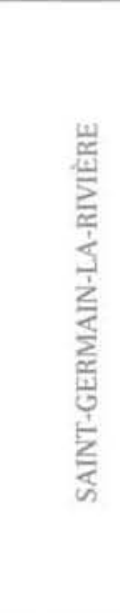 } & 产亩 & $\begin{array}{l}\text { écartement e }(\mathrm{m}) \\
\text { compacité } 1 / \mathrm{e}\left(\mathrm{m}^{-1}\right) \\
\text { Jongueur }(\mathrm{m}) \\
\text { orientation }\left({ }^{\circ}\right)\end{array}$ & $\begin{array}{l}0,00 \\
0,20 \\
0,50 \\
\mathrm{~N} 2\end{array}$ & $\begin{array}{l}0.70 \\
6,00 \\
0.70 \\
N 19\end{array}$ & $\begin{array}{r}4,10 \\
37,40 \\
1,70 \\
\mathrm{~N} 33\end{array}$ & $\begin{array}{l}1,00 \\
8,90 \\
0,20 \\
9,05\end{array}$ & 19 \\
\hline & 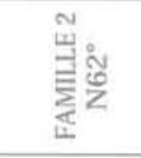 & $\begin{array}{l}\text { écartement e }(\mathrm{m}) \\
\text { compacité } 1 / \mathrm{e}\left(\mathrm{m}^{-1}\right) \\
\text { longueur }(\mathrm{m}) \\
\text { orientation }\left({ }^{\circ}\right)\end{array}$ & $\begin{array}{l}0,00 \\
0,30 \\
0,50 \\
N 37\end{array}$ & $\begin{array}{r}0,40 \\
31,20 \\
1,50 \\
\text { N62 }\end{array}$ & $\begin{array}{r}3,10 \\
548,50 \\
5,20 \\
N 79\end{array}$ & $\begin{array}{r}0,60 \\
108,20 \\
1,10 \\
12,0\end{array}$ & 72 \\
\hline & 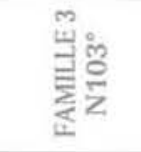 & $\begin{array}{c}\text { écartement e }(\mathrm{m}) \\
\text { compacité } 1 / \mathrm{e}\left(\mathrm{m}^{-1}\right) \\
\text { longueur }(\mathrm{m}) \\
\text { orientation }\left({ }^{\circ}\right)\end{array}$ & $\begin{array}{l}0,00 \\
0,30 \\
0,40 \\
\text { N81 }\end{array}$ & $\begin{array}{r}0,20 \\
52,50 \\
1,70 \\
\text { N103 }\end{array}$ & $\begin{array}{r}3,40 \\
645,50 \\
7,40 \\
\mathrm{~N} 142\end{array}$ & $\begin{array}{r}0,40 \\
107,60 \\
1,20 \\
13,11\end{array}$ & 142 \\
\hline & 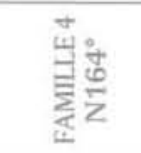 & $\begin{array}{l}\text { écartement e }(\mathrm{m}) \\
\text { compacité } 1 / \mathrm{e}\left(\mathrm{m}^{-1}\right) \\
\text { longueur }(\mathrm{m}) \\
\text { orientation }\left(^{\circ}\right)\end{array}$ & $\begin{array}{l}1,30 \\
0,20 \\
0,50 \\
N 151\end{array}$ & $\begin{array}{l}3,10 \\
0,40 \\
0,50 \\
N 164\end{array}$ & $\begin{array}{r}5,10 \\
0,80 \\
0,60 \\
N 174\end{array}$ & $\begin{array}{r}1,80 \\
0,20 \\
0,10 \\
37,30\end{array}$ & 5 \\
\hline
\end{tabular}
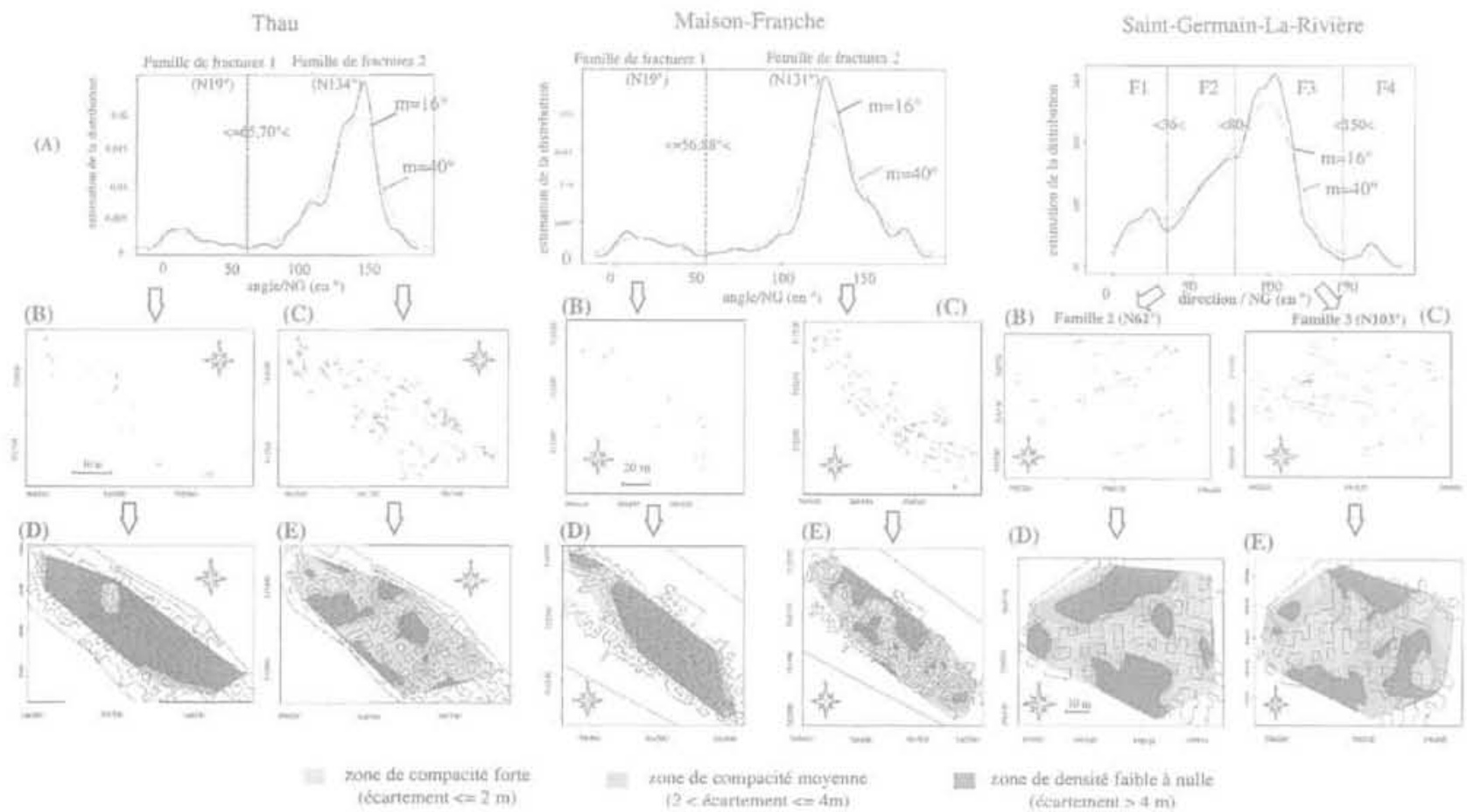

(D)

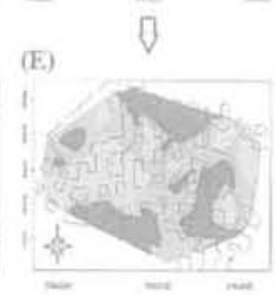

FG.7 Familles de fractures (A, B, C) et cartes de compacité de fractures (D, E) des carrières de Thau, Maison-Franche et Saint-Germain-La-Rivière ( $\mathrm{m}$; taille de la fenêtre de filtrage).

Fracture families (A, B, C) and their density maps (D, E) in quarries: Thau, Maison-Franche and Saint-Germain-La-Riviere ( $\mathrm{m}$ : window size of filtring). 
L'analyse comparative des cartes de compacité totale de fractures met en évidence deux résultats :

a) les fractures s'organisent sous forme de faisceaux dont l'orientation est celle des familles de fractures. A Thau niveau II, les fractures sont orientées $\mathrm{N} 134^{\circ}$ et $\mathrm{N} 19^{\circ}$. Les fractures $\mathrm{N} 134^{\circ}$ sont plus développées que les fractures $\mathrm{N} 19^{\circ}$. Ces deux zones de compacité de fracturation élevée délimitent une zone à faible compacité de fracturation (Fig. 7);

b) le passage des zones de compacité de fractures élevée aux zones de compacité de fractures moyenne puis aux zones de compacité de fractures faible correspond dans la plupart des cas, à une décroissance progressive de la longueur apparente moyenne des fractures. Ainsi, à Saint Germain-LaRivière, la longueur apparente moyenne des fractures est deux fois plus grande dans les zones à faible compacité que dans les zones à forte compacité forte (Fig. 7).

\section{2}

\section{Relation entre les fractures géologiques et les fractures mécaniques}

Dans les carrières de Thau niveau II et MaisonFranche niveau II, il existe une bonne corrélation entre la courbe de distribution de la direction des fractures géologiques karstifiées ou non et celle des fractures mécaniques (Fig. 8). Dans la famille de fractures N19\%, le décalage est de 10 à $15^{\circ}$ entre les fractures géologiques et les fractures mécaniques. Par conséquent, l'orientation des fractures géologiques semble avoir influencé sensiblement l'orientation des fractures mécaniques dans ces deux sites.

Dans les deux carrières, l'orientation de la famille de fractures $\mathrm{N} 131^{\circ}$ correspond à l'orientation des galeries principales. La répartition des fractures mécaniques dépend logiquement de la répartition des contraintes mécaniques dans le toit, celle-ci dépend bien sưr de la gèométrie des carrières, dont en particulier l'orientation des couloirs. On constate également que la densité des fractures mécaniques est fortement liée à la valeur des contraintes verticales dans les sites étudiés. Ainsi, à Thau niveau II l'estimation de la contrainte verticale moyenne atteint la valeur la plus forte de $0,6 \mathrm{MPa}$ : la fracturation mécanique y est la plus importante. C'est aussi en particulier dans la partie Est de ce site que les contraintes mécaniques atteignent les valeurs maximales : les fractures mécaniques y sont aussi concentrées (Fig. 6).

\section{3}

\section{Variation de la connexité des fractures}

En considérant la fréquence des fractures et la densité totale de fractures, les fractures non sécantes représentent plus de $60 \%$ des fractures à Thau niveau Il et à Maison-Franche niveau II et en moyenne $40 \%$ des fractures à Saint-Germain-La-Rivière (Figs. 9, 10 et 11). La connexité ne dépasse pas 3 alors que la connexité égale à 2 est la plus représentée. La proportion des fractures de connexité 1 et 3 varie peu d'un site à l'autre quelle que soit la fréquence des fractures sécantes :

$-50 \%$, à à Saint-Germain-La-Rivière ;

-20 à $25 \%$, à Thau niveau II et Maison-Franche.

Les fractures de connexité élevée apparaissent essentiellement dans les zones de compacité forte des fractures non connexes (Fig. 11).

\section{8}

\section{Conclusion}

Plus de 1000 fractures réparties dans les trois carrières ont été relevées in situ, cartographiées et numérisées. Ces fractures affectent le calcaire oligocène d'Aquitaine dit «calcaire à astéries». Elles ont été distinguées de deux origines, géologique et mécanique. L'analyse de ces fractures est réalisée en considérant la répartition spatiale des paramètres géométriques tels que: ouverture, longueur, direction, orientation des fractures, distribution des familles de fractures et leur écartement.
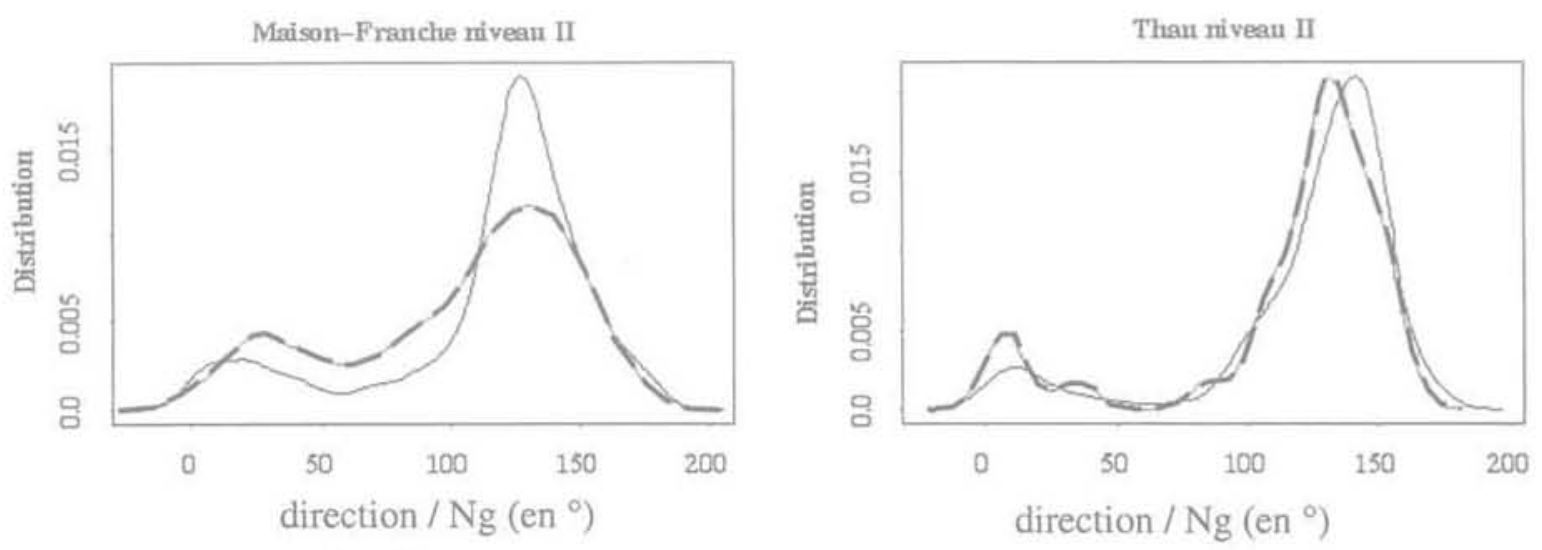

Legende :

Fracturation géologique

- Fracturation mécanique

FIG. Relation entre les directions des fractures géologiques et mécaniques dans les sites étudiés. Relationship between directions of geological fractures and mecanic fractures in different sites. 


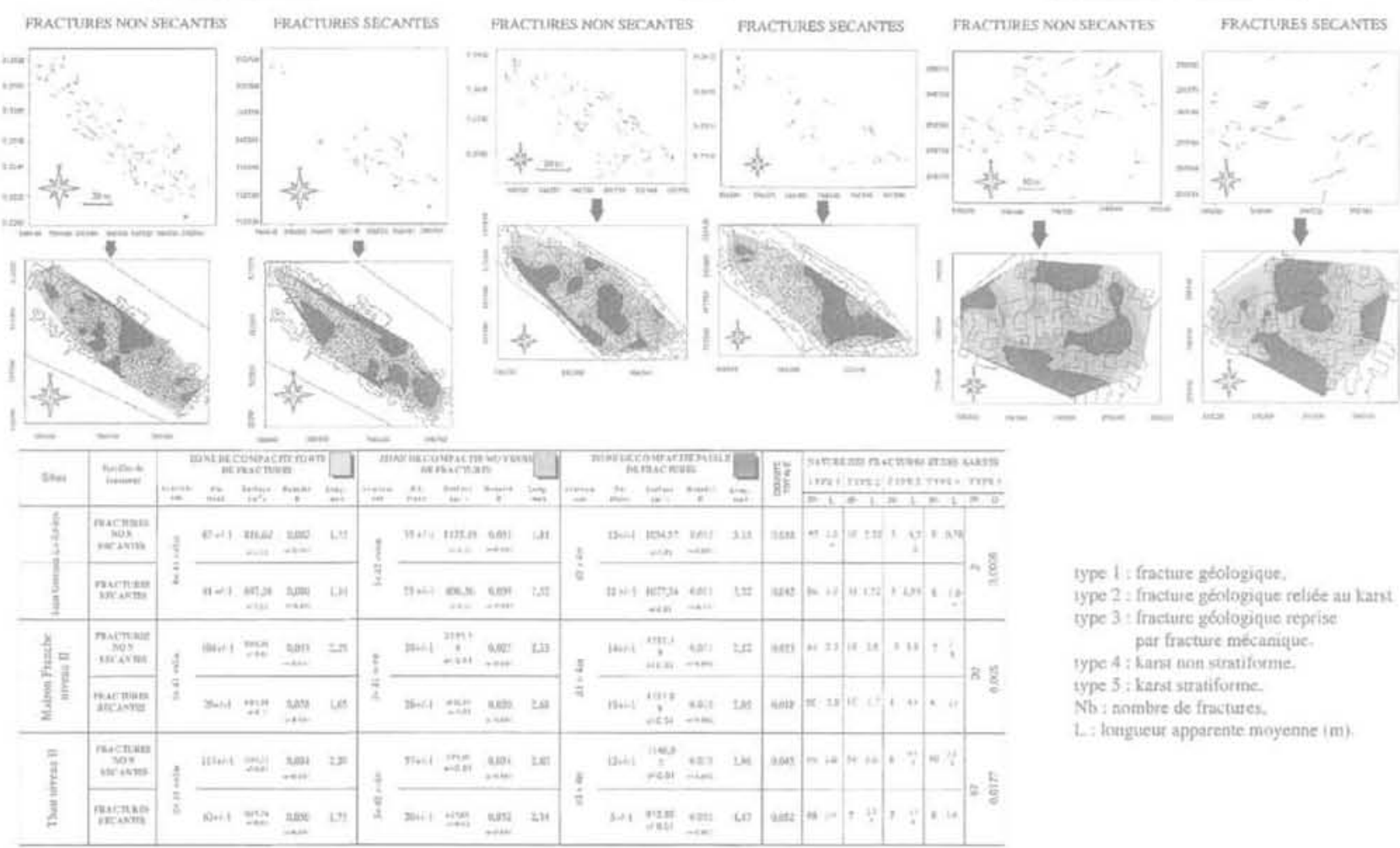

FG. 9 Répartition, composition et cartographie des fractures sécantes et non sécantes dans les sites étudiés. Distribution, composition and mapping of connected and non connected fractures in studied sites.

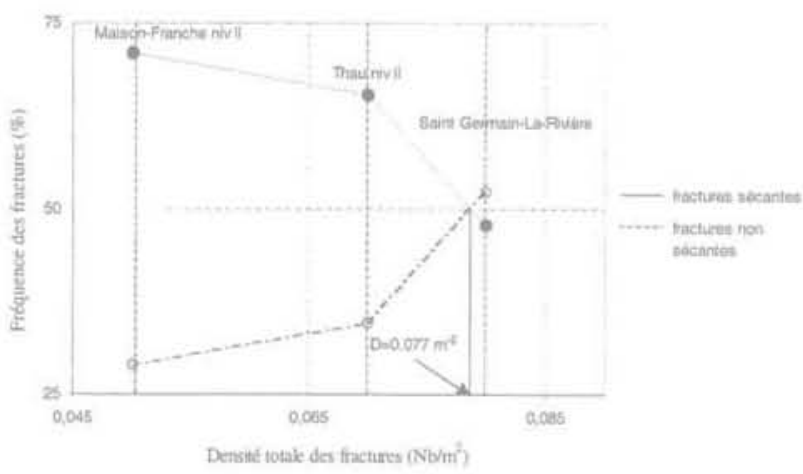

FG. 10 Relation entre la densité totale des fractures (géologique et mécanique) et la fréquence des fractures connexes et non connexes.

Relationship between total density of fractures (geological and mechanical fractures) and frequency of connected fractures and non connected fractures.

En utilisant l'analyse numérique par Systèmes d'information géographique (SIG) en mode image, nous avons établi des cartes de compacité de fractures de même famille ou de familles différentes selon un zonage à trois niveaux : zone de forte compacité, de compacité moyenne et de faible compacité. Pour chaque zone les paramètres suivants ont été extraits : origine, nombre de fractures, écartement, longueur moyenne, fréquence et densité surfacique. Par ailleurs, la connexité des fractures a été calculée et analysée en distinguant «les fractures non sécantes $»$ et «les fractures sécantes ». Pour chacune de ces «familles» de fractures, les paramètres suivants ont été calculés : degré de connexité, fréquence, longueur moyenne, densité surfacique et carte de compacité. Les résultats montrent une relation entre les paramètres des fractures gẻologiques «anciennes ) et les paramètres des fractures mécaniques « récentes $»$ (en particulier la répartition et la géométrie) et, donc un ròle des fractures géologiques dans la stabilité des carrières. Dans les cartes de compacité, les fractures s'organisent dans des champs de compacité forte dont l'orientation est celle des familles principales. Le passage des zones de forte compacité aux zones de compacité faible est accompagné par une décroissance progressive de la longueur moyenne des fractures. Les fractures de connexité élevée apparaissent essentiellement dans les zones de compacité forte des fractures non connexes.

Il est possible, ainsi, d'établir une cartographie précise de la fracturation naturelle, avec ou sans karstification, et de la fracturation anthropique et d'analyser leurs relations mutuelles. Cette analyse devait permettre la mise en évidence de zones anomaliques susceptibles d'être le siége d'effondrements anciens et surtout potentiels. 


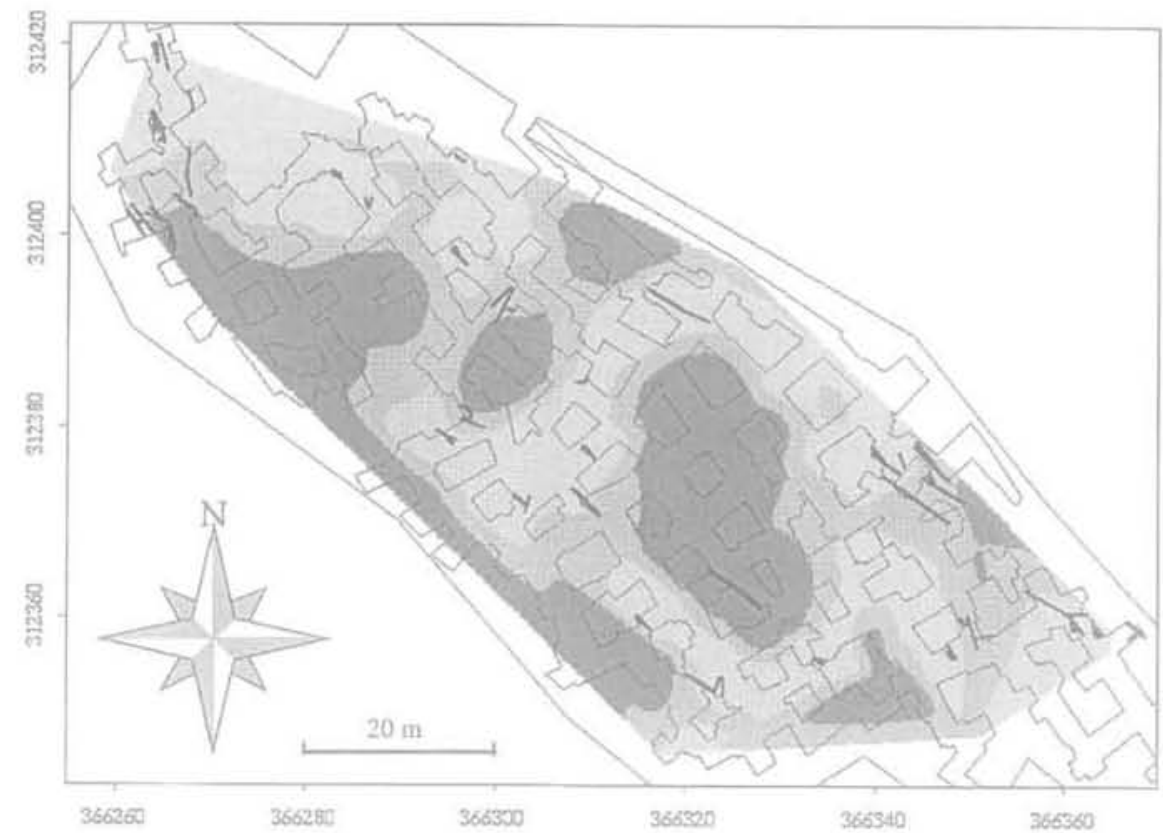

LEGENDE :

Compacité des fractures non connexes :

zone de compacité forte

zone de compacité moyenne

tone de compacité faible

fracture connexe :

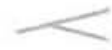

FG. 11 Fractures non connexes et connexes dans la carrière de Thau niveau II. Non connected fractures and connected fractures in quarries of Thau.

\section{Bibliographie}

Allard J.F. - Mẻthode adoptée dans le SudOuest de la France pour létude de la stabilité des carrières souterraines. These de doctorat de $3^{r}$ cycle. Bordeaux 1. $1975,171 \mathrm{p}$.

Blès J.L., Feuga B, - La fracturation des roches : 1. notions de mécanigue des roches ; 2 les fractures naturelles, observation et interprétation. BRGM, série "Manuels et méthodes 1. n 1. 1981. $123 \mathrm{p}$.

Blès J.I. Gros Y, - w l a fracturation du cranite de Bassiès (Pyrénées ariégeoises. Francel. Chronologie des phases tecteniques, évolution des fractures 1 . Bull. Soc. Géol. France, tome 22, $n^{2} 3,1980$. p. $377-390$

Beaufrère C. - Jnfluence des facteurs géologiques et mécaniques sur l'évolution de la stabilité de quatre carrières souterraines exploitant le calcaire oligocène en Gironde. Thèse de doctorat d'Etat, Université de Bordeaux 3, 1986, $407 \mathrm{p}$.

Bodin J.. Razack. M. - " L'analyse d'image appliquée au traîtement automatique de champs de fractures. Propriétés géométriques et lois d'êchelles 4. Bull. Soc. Géol. France, n 4. 1999, p. 579-593.
Bourgine B., Chiles J.P., Castaing C. "Simulation d'un réseau de fractures par un modèle probabilistique hiérarchique n. Ecole nationale supérieure des mines de Paris, Cahiers de gẽostatistique, 1995. p. 81-96.

Brière G., Razack M. - it Mèthode informatique pour l'étude sur clichés aériens de la fracturation des magasins aquifères fissurés n. Rev, Géol, Dyn, Géogr, Phys., 232, 1982, p. 131-142.

Cerepi A. - Milieu poreux matriciel, fracfures ef teneur en eau d'un calcaire en zone de diagenése météorique : calcaire à astéries apierre de Borcieaux n, Oligocène (France). Thèse de doctorat de 3 cycle, Université de Bordeaux 3, 1997. $380 \mathrm{p}$

Collet C. - Systémes d'information géographique en mode image. Lausanne, Presses polytechniques et universitaires romandes, 1992, $182 \mathrm{p}$.

Delay F., Bracq P. - " A vectorial method for the study of the spatial distribution of morphological features applied to needs of hydrogeology in. Computers and Geosciences, 19, 1993, p. 965-980,

Dutartre P. - Etude de la fracturation du granite de la Margeride (règion de Saint-
Alban-sur-Limagnole, Lozère), Gêométrie, cinématique, densité des fractures. Thèse de doctorat de $3^{e}$ cycle, Université de Paris 6, 1982, $344 \mathrm{p}$.

Jourde H. Bicleaux P. Pistre S. - « Modélisation des écoulements en réseaux de fractures orthogonales : influence de la localisation du puits de pompacie sur les rabattements i. Bull. Soc. Géol. France, t. 169, $n^{\circ} 5.1998$, p. 635-644.

Mani J. Broquet P. Ramproux N. - "Rồle de la fracturation dans la détermination de la conductivite hydratiligue des calcaires. Apport des méthodes directes et indirectes o. Bull. Soc. Géol. France, t. $169, n^{\circ}$ 6. 1998, p. 765-773.

Pratviel L. - Essai de cartographie structurale et faciologique du bassin sédimen. taire Ouest Aquitaine pendant I'Oligocène. Thèse de doctorat d'Etat, Université de Bordeaux 1, tomes l et I1. $1972,632 \mathrm{p}$

Ruhiland M. - "Méthodes d'études de la fracturation naturelle des roches associèe à divers modèles structuraux $11 . \mathrm{Sc}$. Géol Bull, 1973, p. 91-113

Saporta G. - Probabilités, analyse des données statistiques. Paris, Technip, 1991. $493 \mathrm{p}$. 